\title{
The PBW property for associative algebras as an integrability condition
}

\author{
BORIS SHOIKHET
}

We develop an elementary method for proving the PoincaréBirkhoff-Witt (PBW) property for associative quadratic-linear algebras, complementary to Drinfeld's results in [D]. The method is very transparent and emphasizes the integrability nature of $\mathrm{PBW}$ property.

We show how the method works in three examples. As a first example, we give a proof of the classical PBW theorem for Lie algebras. As a second, less trivial example, we present a new proof of a result of Etingof and Ginzburg [EG] on PBW property of algebras with a cyclic non-commutative potential in three variables. Finally, as a third example, we found a criterion, for a general quadratic algebra which is the quotient-algebra of $T(V)[\hbar]$ by the two-sided ideal, generated by $\left(x_{i} \otimes x_{j}-x_{j} \otimes x_{i}-\hbar \phi_{i j}\right)_{i, j}$, with $\phi_{i j}$ general quadratic non-commutative polynomials, to be PBW for generic specialization $\hbar=a$. This result seems to be new.

Our condition for PBW property is only sufficient and not necessary, whence the Drinfeld's result in [D, Theorem 2] gives a necessary and sufficient condition. On the other hand, the Drinfeld condition is a countable sequence of equations, and it may be hard to check all of them in practice. Our criterion is a single equation, and is easily checkable, when a particular quadratic-linear algebra fulfils it.

\section{Introduction}

0.1 .

Let $\mathfrak{g}$ be a Lie algebra over a field $\mathrm{k}$ of characteristic 0 . Recall that its universal enveloping algebra is defined as the quotient-algebra

$$
\mathcal{U}(\mathfrak{g})=T(\mathfrak{g}) /(x \otimes y-y \otimes x-[x, y])_{x, y \in \mathfrak{g}}
$$


of the tensor algebra by the two-sided ideal generated by expressions $x \otimes$ $y-y \otimes x-[x, y]$, for all $x, y \in \mathfrak{g}$. The universal enveloping algebra admits a natural ascending filtration $\left\{F_{i}\right\}$, where $F_{i} \subset \mathcal{U}(\mathfrak{g})$ is a k-subspace generated by all monomials of degree $\leq i$ in generators. It is an algebra filtration,

$$
F_{i} * F_{j} \subset F_{i+j}
$$

and one defines the associated graded algebra

$$
\operatorname{gr}_{F} \mathcal{U}(\mathfrak{g})=\oplus_{i \geq-1} F_{i+1} / F_{i}
$$

where $F_{-1}=0$.

The classical Poincaré-Birkhoff-Witt (PBW) theorem says that the algebras $\operatorname{gr}_{F} \mathcal{U}(\mathfrak{g})$ and the symmetric algebra $S(\mathfrak{g})$ are (canonically) isomorphic as graded algebras. Essentially it means that

$$
F_{k} / F_{k-1} \simeq S^{k}(\mathfrak{g})
$$

Note that this PBW theorem can be thought as a consequence of a kind of integrability condition. For example, if an associative algebra $A$ with generators $x_{1}, \ldots, x_{n}$ is defined by relations

$$
x_{i} \otimes x_{j}-x_{j} \otimes x_{i}-\sum_{k} c_{i j}^{k} x_{k}=0
$$

for each pair $1 \leq i<j \leq n$, but the "structure constants" $c_{i j}^{k}$ do not obey the Jacobi identity, the Poincaré-Birkhoff-Witt theorem fails. The corresponding quotient-algebra by the two-sided ideal has a "smaller size" than $S\left(x_{1}, \ldots, x_{n}\right)$.

\section{2 .}

More generally, consider a vector space $V$ over $\mathrm{k}$ with basis $\left\{x_{1}, \ldots, x_{n}\right\}$, consider the tensor algebra $T(V)$, and its $\mathrm{k}[\hbar]$-linear version $T(V)[\hbar]$, where $\hbar$ is a formal parameter. Consider $T(V)[\hbar]$ as a graded algebra, with deg $x_{i}=1$ for $i=1 \ldots n, \operatorname{deg} \hbar=0$. For each pair $1 \leq i<j \leq n$ consider an element $\varphi_{i j} \in \hbar \cdot T(V)[\hbar]$, and consider the quotient-algebra

$$
A=T(V)[\hbar] /\left(x_{i} \otimes x_{j}-x_{j} \otimes x_{i}-\varphi_{i j}\right)_{1 \leq i<j \leq n .}
$$


When $\operatorname{deg}_{x} \varphi_{i j} \leq 2$ for all $i<j$, the algebra $A$ admits the ascending (PBW-like) filtration $\left\{F_{k}\right\}$ where $F_{k}$ is spanned by the monomials of total degree $\leq k$ in $\left\{x_{i}\right\}$. The following problem arises naturally:

Is it true, for a particular algebra $A$ of type (0.1), that for any $k \geq 0$ one has $F_{k+1} / F_{k}=S^{k}\left(x_{1}, \ldots, x_{n}\right)[\hbar]$ as a $\mathrm{k}[\hbar]$-module?

We call this property the Poincaré-Birkhoff-Witt, or shortly PBW, property.

The PBW property of associative algebras has been extensively studied in the literature, see e.g. [D], [PP], [BG], [G], [Sh1, Sh2].

In practice, it may be very difficult to check whether an algebra $A$ of type (0.1) is a PBW algebra. In this paper, we suggest a very general method for proving the PBW property, which emphasizes the "integrability" nature of the PBW condition.

An algebra $A$ of type (0.1) admits, along with the ascending filtration $\left\{F_{k}\right\}$, the following descending filtration $\left\{\Gamma_{k}\right\}$ :

$$
\Gamma_{k}=\hbar^{k} \cdot A
$$

It is an algebra filtration as well, and the associated graded algebra is defined:

$$
\operatorname{gr}_{\Gamma} A=\oplus_{i \geq 0} \Gamma_{i} / \Gamma_{i+1}
$$

Our first Theorem (1.6) shows that, provided some PBW-like property for the descending filtration $\Gamma$ is fulfilled, the algebra $A$ is a PBW algebra, for all but a countable number of specializations at $a \in \mathrm{k}$.

Note that the first quotient $\Gamma_{0} / \Gamma_{1}$ is isomorphic to $S(V)$, for any $\left\{\varphi_{i j}\right\}$.

The $P B W$-like property for the descending filtration $\Gamma$ reads:

$$
\text { For all } k \geq 0 \text {, one has } \Gamma_{k} / \Gamma_{k+1} \simeq \hbar^{k} \cdot S(V)
$$

as $S(V)=\Gamma_{0} / \Gamma_{1}$-module.

On the other side, we develop a method for proving (0.4), which hopefully may work in many cases, in our second Theorem 2.3.

It is instructive to consider what happens with $\operatorname{gr}_{\Gamma} A$ for our "not-a-Liealgebra" example, that is, for the relations

$$
x_{i} \otimes x_{j}-x_{j} \otimes x_{i}-\hbar \sum_{k} c_{i j}^{k} x_{k}
$$

where $\left\{c_{i j}^{k}\right\}$ fail to satisfy the Jacobi identity, and $c_{i j}^{k}=-c_{j i}^{k}$. 
It is easy to see that $\Gamma_{0} / \Gamma_{1} \simeq S(V)$ and $\Gamma_{1} / \Gamma_{2} \simeq \hbar \cdot S(V)$. However, suppose $\left\{c_{i j}^{k}\right\}$ do not satisfy the Jacobi identity:

$$
\sum_{a}\left(c_{i j}^{a} c_{a k}^{b}+c_{j k}^{a} c_{a i}^{b}+c_{k i}^{a} c_{a j}^{b}\right) \neq 0
$$

for some $i, j, k$ and for some $b$.

An easy computation shows that

$\left[\left[x_{i}, x_{j}\right], x_{k}\right]+\left[\left[x_{j}, x_{k}\right], x_{i}\right]+\left[\left[x_{k}, x_{i}\right], x_{j}\right]=\hbar^{2} \sum_{a, b}\left(c_{i j}^{a} c_{a k}^{b}+c_{j k}^{a} c_{a i}^{b}+c_{k i}^{a} c_{a j}^{b}\right) \cdot x_{b}$,

where $[x, y]:=x * y-y * x$.

The left-hand side (l.h.s.) of (0.7) vanishes for any associative algebra. Therefore, the right-hand side (r.h.s.) of $(0.7)$ is a linear in $\left\{\hbar^{2} x_{s}\right\}$ element in $A$ which is 0 in the quotient-algebra. This element belongs to $\Gamma_{2}$ and defines 0 in $\Gamma_{2} / \Gamma_{3}$. Therefore,

$$
\Gamma_{2} / \Gamma_{3}=\hbar^{2} \cdot\left(S(V) /\left(\sum_{a, b}\left(c_{i j}^{a} c_{a k}^{b}+c_{j k}^{a} c_{a i}^{b}+c_{k i}^{a} c_{a j}^{b}\right) \cdot x_{b}\right)_{i, j, k}\right)
$$

is the quotient by the ideal generated by the r.h.s. of $(0.7)$ for all $i, j, k$, and the descending PBW property fails even for $n=2$.

\section{3 .}

This paper is a further development of some ideas of our earlier preprint [Sh2]. Along with a more clear exposition of ideas of [Sh2], this paper contains the following new results.

(1) We upgrade Lemma 2.2 (which is quoted from [Sh2]) to a more powerful Theorem 2.3; roughly speaking, now it is not necessary to perturb all the components of the differential, but only the two most extreme ones, $d\left(\xi_{i j}\right)$ and $d\left(\xi_{i j k}\right)$ (among which the component $d\left(\xi_{i j}\right)$ is given automatically by non-commutative polynomials $\left\{\varphi_{i j}\right\}$ in (1.1)).

(2) We give an application of our method to a new proof of results of Etingof-Ginzburg [EG], on the PBW property of non-commutative algebras "with cyclic cubic potential", for generic parameters. Our proof is contained in Section 3.2. 
(3) As another application, we study the case of a "general" quadratic algebra

$$
\varphi_{i j}=\hbar \sum_{a, b} \alpha_{i j}^{a b} x_{a} \otimes x_{b}
$$

with $\alpha_{i j}^{a b} \in \mathrm{k}$, where $\alpha_{i j}^{a b}=-\alpha_{j i}^{a b}$ but in general $\alpha_{i j}^{a b} \neq \alpha_{i j}^{b a}$. Consider the algebra $A_{\alpha}$ defined as the quotient algebra

$$
A_{\alpha}=T(V)[\hbar] /\left(x_{i} \otimes x_{j}-x_{j} \otimes x_{i}-\varphi_{i j}\right)_{i, j}
$$

We prove that this algebra is $\mathrm{PBW}$ for generic specialization $\hbar=a \in \mathrm{k}$, if the following identity holds: for any $i, j, k, b, c, d$

$$
\mathrm{Cycl}_{i, j, k} \sum_{s}\left(\alpha_{j k}^{s b} \alpha_{i s}^{c d}+\alpha_{j k}^{c s} \alpha_{i s}^{d b}\right)=0
$$

Note that (0.10) implies the Poisson condition

$$
\mathrm{Cycl}_{i j k} \mathrm{Symm}_{a b c} \sum_{s} \beta_{i s}^{a b} \beta_{j k}^{s c}=0
$$

for the bivector $\beta=\sum_{i j} \beta_{i j} \partial_{i} \wedge \partial_{j}$, where

$$
\beta_{i j}=\sum_{a, b}\left(\alpha_{i j}^{a b}+\alpha_{i j}^{b a}\right) x_{a} x_{b} .
$$

\section{4 .}

Drinfeld's beautiful result [D, Theorem 2] gives an explicit criterion which is sufficient and necessary for a formal quadratic deformation of a polynomial algebra $S(V)$ to be a PBW algebra. This criterion reads as a countable number of equations, as follows.

Drinfeld considers an algebra of the form

$$
A=T(V)[[\hbar]] /\left(x_{i} \otimes x_{j}-x_{j} \otimes x_{i}-\varphi_{i j}(\hbar)\right)_{1 \leq i<j \leq n},
$$

where

$$
\varphi_{i j}(\hbar)=\sum_{k, \ell} \varphi_{i j}^{k \ell}(\hbar) x_{k} \otimes x_{\ell}
$$


with

$$
\varphi_{i j}^{k \ell}(0)=0, \quad \varphi_{i j}^{k \ell}=-\varphi_{j i}^{k \ell}, \quad \varphi_{i j}^{k \ell}=\varphi_{i j}^{\ell k}
$$

That is, $\varphi_{i j}^{k \ell}(\hbar)$ are formal power series vanishing at $\hbar=0$, and symmetric in upper indices. (In our treatment, $\varphi_{i j}(\hbar)=\hbar \varphi_{i j}$ is linear in $\hbar$, which is essentially weaker, although our methods can be adjusted for the more general case as well; and we do not assume the symmetry of $\varphi_{i j}(\hbar)$ in upper indices, and work over polynomials rather than formal power series).

Let $V=\left\{x_{1}, x_{2}, \ldots\right\}$ be the space of generators. Drinfeld defines a matrix

$$
B: V \otimes V \otimes V \rightarrow V \otimes V \otimes V
$$

whose entries are

$$
b_{i_{1} i_{2} i_{3}}^{j_{1} j_{2} j_{3}}(\hbar)=\sum_{s} \varphi_{i_{1} s}^{j_{1} j_{2}}(\hbar) \cdot \varphi_{i_{2} i_{3}}^{s j_{3}}(\hbar)
$$

Then Theorem 2 in [D] reads:

For a quadratic algebra (0.11) to be a PBW (graded) algebra the following condition is necessary and sufficient. Denote

$$
A(\hbar)=B(\hbar)\left(1-\frac{1}{3} B(\hbar)\right)^{-1}
$$

and by $a_{i_{1} i_{2} i_{3}}^{j_{1} j_{2} j_{3}}(\hbar)$ its entries. Then for any $i_{1}, i_{2}, i_{3}$ and $j_{1}, j_{2}, j_{3}$

$$
\operatorname{Alt}_{i_{1} i_{2} i_{3}} \operatorname{Sym}_{j_{1} j_{2} j_{3}} a_{i_{1} i_{2} i_{3}}^{j_{1} j_{2} j_{3}}(\hbar)=0 .
$$

For any fixed $i_{1}, i_{2}, i_{3}$ and $j_{1}, j_{2}, j_{3}$ it is a countable number of equations, one for each power of $\hbar$.

Comparing with our result in Theorem 4.1 (formulated as item (3) in Section 0.3 above) we deduce:

Corollary 0.1. Suppose that in (0.9) one additionally has $\alpha_{i j}^{a b}=\alpha_{i j}^{b a}$, and suppose that in (0.12) one has $\varphi_{i j}^{k \ell}(\hbar)=\hbar \cdot \alpha_{i j}^{k \ell}$. Then our equation (0.10) implies all Drinfeld's equations (0.17), for all powers of $\hbar$.

We do not know whether it can be seen directly, by algebraic manipulations with matrices. In our approach, (0.9) gives a sufficient condition for PBW property, by Theorem 4.1, whence the Drinfeld's condition(s) (0.17) are necessary and sufficient. 


\section{General theory}

\subsection{The PBW property}

Throughout the paper, $\mathrm{k}$ is a field of characteristic 0 . All results of the paper (being suitably formulated) hold when the ground field $k$ has an arbitrary characteristic. They can be proven in this generality by literally the same methods. However, one should be careful with the definitions of the symmetric and external powers, defining them as the graded components of the corresponding quotient-algebras, and then to check the exactness of the free dg algebra (Koszul) resolution of the algebra $S(V)$; see Section 2.1. We only consider the characteristic 0 case below mainly to simplify the exposition and to avoid extra technical details.

Let $V=\left\{x_{1}, \ldots, x_{n}\right\}$ be a finite-dimensional vector space over $\mathrm{k}$ with basis $\left\{x_{1}, \ldots, x_{n}\right\}, n=\operatorname{dim}_{k} V$. Denote by $T(V)$ the free associative algebra over k with generators $V$, it is identified with the tensor algebra $T\left(x_{1}, \ldots, x_{n}\right)$ in variables $x_{1}, \ldots, x_{n}$.

Let $\hbar$ be a formal parameter. For any pair $(i, j), 1 \leq i<j \leq n$, choose an element $\varphi_{i j} \in \hbar \cdot T(V)[\hbar]$. Suppose that $\operatorname{deg}_{x} \varphi_{i j} \leq 2$. The main object of our study is the associative algebra

$$
A=T(V)[\hbar] /\left(x_{i} \otimes x_{j}-x_{j} \otimes x_{i}-\varphi_{i j}\right)_{1 \leq i<j \leq n}
$$

which is the $\mathbf{k}[\hbar]$-linear quotient-algebra of the free algebra $T(V)[\hbar]$ by the two-sided ideal, generated by

$$
x_{i} \otimes x_{j}-x_{j} \otimes x_{i}-\varphi_{i j}
$$

for all $1 \leq i<j \leq n$. We set also $\varphi_{i j}=-\varphi_{j i}$ if $i>j$, and $\varphi_{i i}=0$.

With our condition $\operatorname{deg}_{x} \varphi_{i j} \leq 2$, the algebra $A$ is filtered with an ascending filtration $\left\{F_{k}\right\}$. By definition, $F_{k}$ is the image under the natural projection $p: T(V)[\hbar] \rightarrow A$ of the vector space $W_{k} \subset T(V)[\hbar]$ spanned by monomials whose total degree by all $\left\{x_{i}\right\}$ is $\leq k$. It is an algebra filtration, that is

$$
F_{i} * F_{j} \subset F_{i+j}
$$

where $*$ is the product in $A$. 
It allows us to consider the associated graded algebra

$$
\operatorname{gr}_{F} A=\oplus_{i \geq-1} F_{i+1} / F_{i}
$$

where by definition $F_{-1}=0$.

Definition 1.1. The associative algebra $A$ defined in (1.1) is said to satisfy the $P B W$ property if there is a graded $\mathrm{k}[\hbar]$-linear isomorphism

$$
\bigoplus_{\ell \geq 0} F_{\ell} A / F_{\ell-1} A \simeq S(V)[\hbar]
$$

such that each consecutive quotient $F_{\ell} / F_{\ell-1}$ is $\mathrm{k}[\hbar]$-linearly isomorphic to the $\ell$ th symmetric power $S^{\ell}(V)[\hbar]$.

Remark 1.2. We do not require a graded $\mathrm{k}[\hbar]$-linear algebra isomorphism in the above definition, having in mind the case when $\operatorname{deg} \varphi_{i j}=2$ (for some $i, j)$. When $\operatorname{deg} \varphi_{i j} \leq 1$ for all $i, j$, the existence of a linear isomorphism implies the existence of an algebra isomorphism.

A closely related property is the PBW property for algebras over $k$. In our study, these algebras will appear as the specializations of $\mathrm{k}[\hbar]$-linear algebras.

Let $V=\left\{x_{1}, x_{2}, \ldots\right\}$, and let $\psi_{s} \in T(V)$ be non-commutative polynomials of degree $\leq 2$. Consider the associative algebra

$$
B=T(V) /\left(\psi_{s}\right)
$$

The algebra $B$ is endowed with natural ascending filtration (which is denoted also by $F$ ), where $F_{k}$ is formed by the elements of degree $\leq k$ in $\left\{x_{i}\right\}$. Denote $F_{-1}=0$.

Definition 1.3. The associative algebra $B$ defined in (1.6) is said to satisfy the PBW property if there is an isomorphism of graded vector spaces:

$$
\bigoplus_{\ell \geq 0} F_{\ell} / F_{\ell-1} \simeq S(V)
$$

The following Lemma is very elementary. We include it here for further references. 
Lemma 1.4. Let $A$ be a $\mathrm{k}[\hbar]$-algebra as in (1.1). Suppose the algebra $A$ satisfies the $\mathrm{k}[\hbar]$-linear $P B W$ condition of Definition 1.1 . Then for an $a \in \mathrm{k}$, the specialization

$$
A_{a}:=A \otimes_{\mathrm{k}[\hbar]} \mathrm{k}[\hbar] /(\hbar-a)
$$

is an algebra $B$ as in (1.6), with

$$
\psi_{i j}=x_{i} \otimes x_{j}-x_{j} \otimes x_{i}-\varphi_{i j}(a)
$$

Moreover, any specialization $A_{a}, a \in \mathrm{k}$, satisfies the PBW condition of Definition 1.3.

Proof. We need to know that $A$ is a free $\mathrm{k}[\hbar]$-module, which follows from Definition 1.1 , saying that $\operatorname{gr}_{F} A$ is. Now $A$ is a quotient $\mathrm{k}[\hbar]$-module $A=$ $T(V)[\hbar] / I[\hbar]$ where $I$ is the two-sided ideal. As $A$ is a free (therefore, a flat) $\mathrm{k}[\hbar]$-module, the specialization $A_{a}$ is the quotient $T(V)[\hbar]_{a} / I[\hbar]_{a}$ of specializations, whence the first assertion follows. To prove that $A_{a}$ is a $\mathrm{PBW}$ as in Definition 1.3, we first mention that $F_{\ell}(A) / F_{\ell-1}(A)$ is a free $\mathrm{k}[\hbar]$-module, by (1.5). Therefore, $\left(F_{\ell}(A) / F_{\ell-1}(A)\right)_{a} \simeq F_{\ell}(A)_{a} / F_{\ell-1}(A)_{a}$. The r.h.s. is $F_{\ell}(B) /$ $F_{\ell-1}(B)$, with $B=A_{a}$, by the first assertion of Lemma.

\subsection{First main theorem}

The main topic of this paper is a rather general method, which reduces the proving of PBW property to another, more easily checked, property. Let us formulate it.

Along with the ascending filtration $\left\{F_{k}\right\}$, the associative algebra $A$ admits the natural descending filtration $\{\Gamma\}$ by powers of $\hbar$ :

$$
\Gamma_{\ell}=\hbar^{\ell} A \text {. }
$$

It is also an algebra filtration:

$$
\Gamma_{i} * \Gamma_{j} \subset \Gamma_{i+j}
$$

which allows us to define the associated graded algebra

$$
\operatorname{gr}_{\Gamma} A=\oplus_{i \geq 0} \Gamma_{i} / \Gamma_{i+1}
$$


Definition 1.5. The associative algebra $A$ is said to satisfy the $P B W$-like property for the descending filtration $\Gamma$ if $\oplus_{\ell \geq 0} \Gamma_{\ell} A / \Gamma_{\ell+1} A \simeq S(V)[\hbar]$ as a graded $\mathrm{k}[\hbar]$-algebra, such that $\Gamma_{\ell} A / \Gamma_{\ell+1} A \simeq \hbar^{\ell} S(V)$.

Our main result in Section 1 is:

Theorem 1.6. Let $\mathrm{k}$ be a field and let $A$ be as above. Suppose $A$ satisfies the PBW-like property for the descending filtration $\Gamma$, see Definition 1.5. Then:

(1) Suppose that

$$
\bigcap_{\ell \geq 0} \Gamma_{\ell}=0
$$

Then the algebra $A$ obeys the PBW property of Definition 1.5, with respect to the ascending filtration $\left\{F_{k}\right\}$. Its specialization

$$
A_{a}=A \otimes_{\mathrm{k}[\hbar]} \mathrm{k}[\hbar] /(\hbar-a)
$$

is a PBW algebra in the sense of Definition 1.3 and is $T(V) /\left(\psi_{i j}\right)$, with $\psi_{i j}$ as in (1.9), for any $a \in \mathrm{k}$.

(2) Suppose that the vector space $V=\left\{x_{1}, x_{2}, \ldots\right\}$ in (1.1) is finitedimensional over $\mathrm{k}$. Then in general, when $\bigcap_{k \geq 0} \Gamma_{k} \neq 0$, its support $\mathcal{S}$ (as of a $\mathrm{k}[\hbar]$-module) is at most a countable set of points $s \in \mathrm{k}$. For any $a \in \mathrm{k} \backslash \mathcal{S}$, the specialization $A_{a}=A \otimes_{\mathrm{k}[\hbar]} \mathrm{k}[\hbar] /(\hbar-a)$ is a PBW algebra.

Remark 1.7. Equation (1.13) always holds when all $\varphi_{i j}$ are linear in $x_{i}$ 's, see Lemma 3.1 below. If $\left\{\varphi_{i j}\right\}$ are quadratic in $x_{i}$ 's, (1.13) is not true, in general. We show it in the simplest case in Example right below. See also Section 3.2.1 where such phenomenon is shown for Etingof-Ginzburg algebras with cyclic cubic potential.

Example 1.8. Consider the algebra $A=T(x, y)[\hbar] /(x y-y x=\hbar(x y-y x))$. Then $x y-y x \in \Gamma_{\ell}$ for all $\ell \geq 0$, and $\bigcap_{\ell \geq 0} \Gamma_{\ell}$ is the two-sided $T(x, y)$-ideal generated by $x y-y x$.

\subsection{Proof of Theorem $\mathbf{1 . 6}$}

\subsubsection{Lemma on two filtrations.}

Lemma 1.9. Let $R$ be a ring, and $L$ an $R$-module. Suppose that $L$ is endowed with an ascending filtration $F$, and with a descending filtration $\Gamma$. 
Then the filtration $F$ induces an ascending filtration on each consecutive quotient $\operatorname{gr}_{\Gamma}^{i} L$, and the filtration $\Gamma$ induces a descending filtration on each consecutive quotient $\operatorname{gr}_{\Gamma}^{j} L$. Their consecutive quotients are canonically isomorphic:

$$
\operatorname{gr}_{\Gamma}^{i} \operatorname{gr}_{F}^{j} L \simeq \operatorname{gr}_{F}^{j} \operatorname{gr}_{\Gamma}^{i} L
$$

Proof. It is clear, as both $R$-modules are isomorphic to

$$
\Gamma_{i} \cap F_{j} /\left(\Gamma_{i+1} \cap F_{j}+\Gamma_{i} \cap F_{j-1}\right) .
$$

It is clear that the same statement is true when both filtrations are ascending or descending.

1.3.2. Proof of Theorem 1.6(i). We need to prove that, under the assumption of Theorem 1.6, the quotient $F_{k} A / F_{k-1} A$ is $\mathrm{k}[\hbar]$-linearly isomorphic to $S^{k}(V)[\hbar]$. This quotient $F_{k} A / F_{k-1} A$ is filtered by descending filtration $\Gamma$, and by Lemma 1.9

$$
\operatorname{gr}_{\Gamma}^{j}\left(F_{k} A / F_{k-1} A\right) \simeq \operatorname{gr}_{F}^{k} \Gamma_{j} A / \Gamma_{j+1} A \simeq \operatorname{gr}_{F}^{k}\left(\hbar^{k} S(V)[\hbar]\right) \simeq \hbar^{j} S^{k}(V)
$$

as $\mathrm{k}[\hbar]$-modules. (Here in the second equality we used the assumption of Theorem 1.6, on the PBW-like property for the descending filtration $\Gamma$.) As well, we know from these assumptions that $\oplus_{j \geq 0} \operatorname{gr}_{\Gamma}^{j} \operatorname{gr}_{F}^{k} A \simeq S^{k}(V)[\hbar]$ is a free $\mathrm{k}[\hbar]$-module (for a fixed $k)$. Therefore, the $\mathrm{k}[\hbar]$-module $\operatorname{gr}_{F}^{k} A /\left(\left(\cap_{j} \Gamma_{j}\right) \cap\right.$ $\operatorname{gr}_{F}^{k} A$ ) is also free. It follows that the space of its generators (as a $\mathrm{k}[\hbar]$ module) is $\operatorname{gr}_{\Gamma}^{0} \operatorname{gr}_{F}^{k} A \simeq S^{k}(V)$, and then $\operatorname{gr}_{F}^{k} A /\left(\left(\cap_{j} \Gamma_{j}\right) \cap \operatorname{gr}_{F}^{k} A\right)$ is isomorphic to the free $\mathrm{k}[\hbar]$-module $S^{k}(V)[\hbar]$.

Now, as $\bigcap_{j \geq 0} \Gamma_{j}=0$ by assumption of (i), it follows that $F_{k} A / F_{k-1} A \simeq$ $S^{k}(V)[\hbar]$, as an $\mathrm{k}[\hbar]$-module.

The remaining assertions of Theorem 1.6(i) follow now from Lemma 1.4.

1.3.3. The structure of a finitely-generated $\mathbf{k}[\hbar]$-module. Before proving the part (ii) of Theorem, recall the structure theorem for finitely generated $\mathbf{k}[\hbar]$ (resp., $\mathbf{k}[[\hbar]]$ ) modules. This result is well-known, and we refer the reader to the textbooks in Algebra for a proof.

Lemma 1.10. (i) Let $\mathrm{k}$ be any field. Any finitely generated $\mathrm{k}[[\hbar]]$-module is a direct sum

$$
M=M_{\text {free }} \oplus M_{n_{1}} \oplus \cdots \oplus M_{n_{\ell}}
$$


where $M_{\text {free }}$, is a free $\mathrm{k}[[\hbar]]$-module, $n_{i} \in \mathbb{Z}_{>0}$, and

$$
M_{n}=\mathrm{k}[[\hbar]] / \hbar^{n} \mathrm{k}[[\hbar]]
$$

(ii) Let $\mathrm{k}$ be an algebraically closed field. Any finitely generated $\mathrm{k}[\hbar]$-module $M$ is a direct sum

$$
M=M_{\text {free }} \oplus M_{a_{1}, n_{1}} \oplus \cdots \oplus M_{a_{\ell}, n_{\ell}},
$$

where $M_{\text {free }}$ is a free $\mathrm{k}[\hbar]$-module of a finite rank, $a_{i} \in \mathrm{k}, n_{i} \in \mathbb{Z}_{>0}$, and

$$
M_{a, n}=\mathrm{k}[\hbar] /(\hbar-a)^{n} \mathrm{k}[\hbar] .
$$

(iii) When $k$ is not necessarily an algebraically closed field, a finitely generated $\mathrm{k}[\hbar]$-module $M$ is a direct sum

$$
M=M_{\text {free }} \oplus M_{p_{1}(\hbar), n_{1}} \oplus \cdots \oplus M_{p_{\ell}(\hbar), n_{\ell}},
$$

where $M_{\text {free }}$ is a free $\mathrm{k}[\hbar]$-module, $p_{i}(\hbar)$ are (monic) irreducible polynomials in $\mathrm{k}[\hbar], n_{i} \in \mathbb{Z}_{>0}$, and

$$
M_{p(\hbar), n}=\mathrm{k}[\hbar] / p(\hbar)^{n} \mathrm{k}[\hbar] .
$$

Proof. It is standard. Notice that the statement (ii) is nothing but the Jordan normal form theorem, for the case of an algebraically closed field, and the statement (iii) is its direct analogue for general (not necessarily algebraically closed) fields. The modules $M_{n}$ are indecomposable $\mathrm{k}[[\hbar]]$-modules, as well as the modules $M_{a, n}$ (corresp., $M_{p(\hbar), n}$ for irreducible $p(\hbar)$ ) are indecomposable $\mathrm{k}[\hbar]$-modules. Together with the one-dimensional free module, they exhaust the list of finitely generated indecomposable modules over the corresponding commutative $k$-algebras $(\mathrm{k}[[\hbar]]$ and $\mathrm{k}[\hbar])$.

1.3.4. Proof of Theorem 1.6(ii). The same argument as in the proof of Theorem 1.6(i) shows that, in general case,

$$
\operatorname{gr}_{F}^{p}(A) /\left(\operatorname{gr}_{F}^{p}(A) \cap I\right) \simeq S^{p}(V)[\hbar]
$$

for any $k \geq 0$, where

$$
I=\bigcap_{\ell \geq 0} \Gamma_{\ell}(A) .
$$

We consider $I \cap \operatorname{gr}_{F}^{p}(A)$ as a $\mathrm{k}[\hbar]$-module. It is finitely generated for any fixed $p$, as $F_{p}(A)$ is generated by (a finite number) of monomials in $x_{1}, \ldots, x_{n}$ 
of total degree $\leq p$. (Here we use essentially that $\operatorname{dim} V<\infty$, which is an assumption in (ii); otherwise $F_{p}$ would fail to be a finitely generated $\mathrm{k}[\hbar]$ module).

Therefore, Lemma 1.10(iii) is applicable to $M=I \cap \operatorname{gr}_{F}^{p}(A)$. We want to prove that the free component $M_{\text {free }}$ in (1.19) is 0 . It is enough to prove that the specialization $M_{0}$ of $M$ at $\hbar=0$ is 0 . We have: $M_{0}=M \otimes_{\mathrm{k}[\hbar]}(\mathrm{k}[\hbar] /(\hbar))$. It is 0 , as $I \otimes_{\mathrm{k}[\hbar]}(\mathrm{k}[\hbar] /(\hbar))=(\hbar I) \otimes_{\mathrm{k}[\hbar]}(\mathrm{k}[\hbar] /(\hbar))=0$ (we use $\left.I=\hbar I\right)$.

Therefore, for any fixed $p$,

$$
M=I \cap \operatorname{gr}_{F}^{p}(A)=M_{p_{1}(\hbar), n_{1}} \oplus \cdots \oplus M_{p_{\ell}(\hbar), n_{\ell}}
$$

for finite $\ell$.

Its specialization $M_{s}=0$ for any $s \in \mathrm{k}$ which is not a root of either of polynomials $p_{k}(\hbar)$. Denote by $\mathcal{S}$ the set of $s \in \mathrm{k}$ such that $p_{i}(s)=0$ for some $i$.

For any $t \in \mathbf{k} \backslash \mathcal{S}$ one has:

$$
\left(\operatorname{gr}_{F}^{p}(A) /\left(I \cap \operatorname{gr}_{F}^{p}(A)\right)\right)_{t}=\left(\operatorname{gr}_{F}^{p}(A)\right)_{t} /\left(I \cap \operatorname{gr}_{F}^{p}(A)\right)_{t}=\left(\operatorname{gr}_{F}^{p}(A)\right)_{t}
$$

as $\operatorname{gr}_{F}^{p}(A) /\left(I \cap \operatorname{gr}_{F}^{p}(A)\right) \simeq S^{p}(V)[\hbar]$ is a free $\mathrm{k}[\hbar]$-module, and the corresponding $\operatorname{Tor}_{\mathrm{k}[\hbar]}^{1}=0$.

We obtain that for any $t \in \mathrm{k} \backslash \mathcal{S}$

$$
\operatorname{gr}_{F}^{p}(A)_{t} \simeq S^{p}(V)[\hbar] /(\hbar-t) \simeq S^{p}(V)
$$

It remains to prove that $\left(F_{p}(A) / F_{p-1}(A)\right)_{t} \simeq\left(F_{p}(A)\right)_{t} /\left(F_{p-1}(A)\right)_{t}$ for any $t \in \mathrm{k} \backslash \mathcal{S}$. We apply once again Lemma $1.10(\mathrm{iii})$, which says that

$$
F_{p}(A) / F_{p-1}(A)=S^{p}(V)[\hbar] \bigoplus\left(\oplus M_{\theta_{i}(\hbar), m_{i}}\right)
$$

where the second summand has support in $\mathcal{S}$. Then for $t \in \mathbf{k} \backslash \mathcal{S}$, one has

$$
\operatorname{Tor}_{\mathrm{k}[\hbar]}^{1}\left(F_{p}(A) / F_{p-1}(A), \mathrm{k}[\hbar] /(\hbar-t)\right)=0
$$

and we are done.

\section{A way to prove the descending filtration PBW property}

Here we formulate and prove an effective tool allowing (in some cases) to prove that the assumption of Definition 1.5 (and Theorem 1.6) is fulfilled. That is, we establish a way to prove that $\oplus_{j \geq 0} \Gamma_{j} A / \Gamma_{j+1} A \simeq S(V)[\hbar]$ as a graded $\mathbf{k}[\hbar]$-algebra. 


\subsection{The Koszul free resolution of the algebras of polynomials}

Here we recall the construction of a free dg resolution of the polynomial algebra $S(V)$, called the Koszul resolution. The vector space $V$ is either finite-dimensional over $k$, or graded with finite-dimensional graded components.

Denote by $\Lambda^{-}(V)$ the cofree commutative cocommutative coalgebra without counit on the space $V[1]$ (concentrated in degree -1 if $V$ is in degree 0 ). That is, as a vector space, $\Lambda^{-}(V)=S^{+}(V[1])$, and the coproduct is

$$
\begin{aligned}
& \Delta\left(v_{1} \wedge \cdots \wedge v_{\ell}\right) \\
& =\sum_{\substack{\ell=a+b \\
a, b>0}} \sum_{\substack{(a, b) \text {-shuffles } \\
\sigma \in \Sigma_{\ell}}}(-1)^{\sharp \sigma}\left(v_{\sigma_{1}} \wedge \cdots \wedge v_{\sigma_{a}}\right) \bigotimes\left(v_{\sigma_{a+1}} \wedge \cdots \wedge v_{\sigma_{\ell}}\right) .
\end{aligned}
$$

Now shift $\Lambda^{-}(V)$ by 1 to the right, and take the tensor algebra of this graded vector space:

$$
\mathcal{R}^{\bullet}=T\left(\Lambda^{-}(V)[-1]\right) .
$$

Introduce a differential $d$ in $\mathcal{R}^{*}$ (as in a dg algebra, satisfying the Leibniz rule with signs), defining it on generators $\left(v_{i_{1}} \wedge \cdots \wedge v_{i_{k}}\right)[-1]$ by

$$
\begin{aligned}
& d\left(\left(v_{i_{1}} \wedge \cdots \wedge v_{i_{k}}\right)[-1]\right) \\
& \quad=\sum_{i}\left(\Delta_{i}^{1}\left(v_{i_{1}} \wedge \cdots \wedge v_{i_{k}}\right)\right)[-1] \otimes\left(\Delta_{i}^{2}\left(v_{i_{1}} \wedge \cdots \wedge v_{i_{k}}\right)\right)[-1]
\end{aligned}
$$

where

$$
\Delta(\omega)=\sum_{i} \Delta_{i}^{1}(\omega) \otimes \Delta_{i}^{2}(\omega)
$$

is the notation for the coproduct.

The equation $d^{2}=0$ follows from the coassociativity of $\Delta$.

Choose a basis $\left\{x_{1}, \ldots, x_{n}, \ldots\right\}$ in $V$. Denote by $\xi_{1}, \ldots, \xi_{n}, \ldots$ the corresponding elements (the cogenerators) in $\Lambda^{-}(V)$. Then $\operatorname{deg} \xi_{i}=-1$. After the shift by $1, \Lambda^{-}(V)[-1]$, we use the notations $x_{i}$ for $\xi_{i}[-1] \in \mathcal{R}^{0}$. Denote $\xi_{i j}=\xi_{i} \wedge \xi_{j} \in(\mathcal{R})^{-1}$. Then $(2.3)$ gives

$$
\begin{gathered}
d x_{i}=0, \\
d\left(\xi_{i j}\right)=x_{i} \otimes x_{j}-x_{j} \otimes x_{i} .
\end{gathered}
$$


A general element in $(\mathcal{R})^{-1}$ is a sum of the following elements $\omega \in T$ $\left(x_{1}, \ldots, x_{n}, \ldots\right) \otimes \xi_{i j} \otimes T\left(x_{1}, \ldots, x_{n}, \ldots\right)$.

By Leibniz rule: $d(\omega) \in T(V) \otimes\left(x_{i} \otimes x_{j}-x_{j} \otimes x_{i}\right) \otimes T(V)$.

Thus the image $d\left(\mathcal{R}^{\bullet}\right) \subset \mathcal{R}^{0}$ is the two-sided ideal generated by $x_{i} \otimes$ $x_{j}-x_{j} \otimes x_{i}$. Therefore,

$$
H^{0} \mathcal{R}^{\bullet}=S(V)
$$

as an algebra.

In fact, the higher cohomology of $\mathcal{R}^{\bullet}$ vanish.

Lemma 2.1. The higher cohomology $H^{k} \mathcal{R}^{\bullet}=0$ for any $k \leq-1$.

It is standard in theory of Koszul algebras; see e.g., [PP] or [BGS]. In fact, one can construct a free "Koszul" resolution of any Koszul algebra $A$, which coincides with the one we just described for the case $A=S(V)$.

We will often use the formula for $d\left(\xi_{i j k}\right)$ where $\xi_{i j k}=\xi_{i} \wedge \xi_{j} \wedge \xi_{k} \in \mathcal{R}^{-2}$. It can be easily seen from (2.2) that

$$
d\left(\xi_{i j k}\right)=\left[x_{i}, \xi_{j k}\right]+\left[x_{j}, \xi_{k i}\right]+\left[x_{k}, \xi_{i j}\right]
$$

Then applying $d$ once again, we see that

$$
d^{2}\left(\xi_{i j k}\right)=\left[x_{i},\left[x_{j}, x_{k}\right]\right]+\left[x_{j},\left[x_{k}, x_{i}\right]\right]+\left[x_{k},\left[x_{i}, x_{j}\right]\right]
$$

which is 0 by the Jacobi identity.

\subsection{A lemma}

We start with some lemma which is much weaker than Theorem 2.3 below. Nevertheless, it better explains "what goes on", so we decided to keep it here. This lemma is quoted from [Sh2], whence Theorem 2.3 is new.

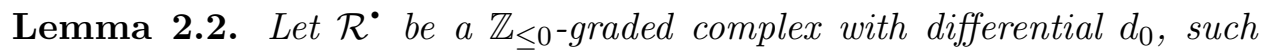
that $H^{i}\left(\mathcal{R}^{\bullet}, d_{0}\right)$ vanishes for all $i \neq 0$.

(i) Consider $\mathcal{R}_{\hbar}^{\cdot}=\mathcal{R}^{\bullet} \otimes \mathrm{k}[\hbar]$. Let $d_{\hbar}: \mathcal{R}_{\hbar}^{\cdot} \rightarrow \mathcal{R}_{\hbar}^{\bullet+1}$ be an $\mathrm{k}[\hbar]$-linear map of degree +1

$$
d_{\hbar}=d_{0}+\hbar d_{1}+\hbar^{2} d_{2}+\cdots \hbar^{n} d_{n}
$$

(a finite sum) such that

$$
d_{\hbar}^{2}=0
$$


Denote by $H_{\hbar}^{\cdot}$ the cohomology of the complex $\left(\mathcal{R}_{\hbar}^{\cdot}, d_{\hbar}\right)$. Consider the filtration $\mathcal{R}_{\hbar}^{\cdot} \supset \hbar \mathcal{R}_{\hbar}^{\cdot} \supset \hbar^{2} \mathcal{R}_{\hbar}^{\cdot} \supset \ldots$, and the induced filtration on $H_{\hbar}^{\cdot}$ : $F_{i} H_{\hbar}^{\bullet}=\operatorname{Im}\left(i: H^{\bullet}\left(\hbar^{i} \mathcal{R}_{\hbar}^{\bullet}, d_{\hbar}\right) \rightarrow H^{\bullet}\left(\mathcal{R}_{\hbar}^{\bullet}, d_{\hbar}\right)\right)$. Then there are canonical isomorphisms of $\mathrm{k}[\hbar]$-modules

$$
F_{i} H_{\hbar}^{0} / F_{i+1} H_{\hbar}^{0} \stackrel{\sim}{\rightarrow} \hbar^{i} H^{0}\left(\mathcal{R}^{\bullet}, d_{0}\right)
$$

and $F_{i} H_{\hbar}^{k} / F_{i+1} H_{\hbar}^{k}=0$ for $k<0$,

(ii) the same statement as in $(i)$, for $\mathcal{R}_{[[\hbar]]}^{\bullet}=\mathcal{R}^{\bullet}[[\hbar]]$, and

$$
d_{\hbar}=d_{0}+\hbar d_{1}+\hbar^{2} d_{2}+\cdots
$$

(possibly an infinite sum), and $d_{\hbar}^{2}=0$.

Proof. Consider the short exact sequences of complexes $S_{k}$ :

$$
0 \rightarrow \hbar^{k+1} \mathcal{R}_{\hbar}^{\cdot} \rightarrow \hbar^{k} \mathcal{R}_{\hbar}^{\cdot} \rightarrow \hbar^{k} \mathcal{R}_{\hbar}^{\cdot} / \hbar^{k+1} \mathcal{R}_{\hbar}^{\cdot} \rightarrow 0
$$

The complex $\hbar^{k} \mathcal{R}_{\hbar}^{\bullet} / \hbar^{k+1} \mathcal{R}_{\hbar}^{\bullet}$ has the differential $d_{0}$ because all its higher components vanish. Consider the long exact sequence of cohomology corresponded to this short exact sequence of complexes. It has many zero terms, namely $H^{\ell}\left(\hbar^{k} \mathcal{R}_{\hbar}^{\cdot} / \hbar^{k+1} \mathcal{R}_{\hbar}^{\bullet}\right)=0$ for $\ell \leq-1$. Then the long exact sequence proves that the imbedding $\hbar^{k+1} \mathcal{R}_{\hbar}^{\bullet} \hookrightarrow \hbar^{k} \mathcal{R}_{\hbar}^{\bullet}$ induces an isomorphism on $\ell$ th cohomology for all $\ell \leq-1$. Consider the end fragment of the long exact sequence:

$$
\begin{aligned}
& \cdots \rightarrow H^{1}\left(\hbar^{k+1} \mathcal{R}_{\hbar}^{\cdot}\right) \rightarrow H^{1}\left(\hbar^{k} \mathcal{R}_{\hbar}^{\cdot}\right) \rightarrow 0 \rightarrow \\
& \rightarrow H^{0}\left(\hbar^{k+1} \mathcal{R}_{\hbar}^{\bullet}\right) \rightarrow H^{0}\left(\hbar^{k} \mathcal{R}_{\hbar}^{\bullet}\right) \rightarrow H^{0}\left(\hbar^{k} \mathcal{R}_{\hbar}^{\bullet} / \hbar^{k+1} \mathcal{R}_{\hbar}^{\bullet}\right) \rightarrow 0
\end{aligned}
$$

It proves all assertions of the Lemma.

\subsection{Second main theorem}

Here we generalize Lemma 2.2 to weaken the assumptions under which the algebra $H^{0}\left(\mathcal{R}_{\hbar}^{\cdot}\right)$ enjoys the PBW property. We remark that in the proof of Lemma 2.2 we did not use that the differential $d_{0}$ can be "perturbed" in all degrees. In fact, all we need is to perturb the differential $d_{0}$ in degrees -1 and -2 , such that $d_{\hbar}^{(-1)} \circ d_{\hbar}^{(-2)}=0$. The differential component $d_{\hbar}^{(-1)}$ is encoded in the non-commutative algebra we check for the PBW property, 
so the only thing we need is to construct $d_{\hbar}^{(-2)}=d_{0}^{(-2)}+\mathcal{O}(\hbar)$, such that

$$
d_{\hbar}^{(-1)} \circ d_{\hbar}^{(-2)}=0
$$

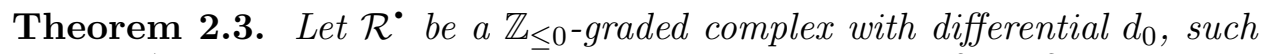
that $H^{-1}\left(\mathcal{R}^{\bullet}\right)$ vanishes (without any assumptions on $\left.H^{-2}, H^{-3}, \ldots\right)$.

(i) Consider $\mathcal{R}_{\hbar}^{\bullet}=\mathcal{R}^{\bullet} \otimes \mathrm{k}[\hbar]$. For $\ell=-1$ and $\ell=-2$, let $d_{\hbar}^{(\ell)}: \mathcal{R}_{\hbar}^{\bullet} \rightarrow \mathcal{R}_{\hbar}^{\bullet+1}$ be an $\mathrm{k}[\hbar]$-linear map of degree +1

$$
d_{\hbar}^{(\ell)}=d_{0}^{(\ell)}+\hbar d_{1}^{(\ell)}+\hbar^{2} d_{2}^{(\ell)}+\cdots \hbar^{n} d_{n}^{(\ell)}
$$

(a finite sum) such that

$$
d_{\hbar}^{(-1)} \circ d_{\hbar}^{(-2)}: \mathcal{R}_{\hbar}^{-2} \rightarrow \mathcal{R}_{\hbar}^{0}=0
$$

Denote by $\overline{\mathcal{R}}_{\hbar}$ the following complex:

$$
0 \rightarrow \mathcal{R}_{\hbar}^{-2} \stackrel{d_{\hbar}^{(-2)}}{\longrightarrow} \mathcal{R}_{\hbar}^{-1} \stackrel{d_{\hbar}^{(-1)}}{\longrightarrow} \mathcal{R}_{\hbar}^{0} \rightarrow 0
$$

and denote by $H_{\hbar}^{\ell}, \ell=0,-1,-2$, its cohomology.

Consider the filtration $\overline{\mathcal{R}}_{\hbar} \supset \hbar \overline{\mathcal{R}}_{\hbar} \supset \hbar^{2} \overline{\mathcal{R}}_{\hbar} \supset \cdots$, and the induced filtration on $H_{\hbar}^{\ell}: F_{i} H_{\hbar}^{\ell}=\operatorname{Im}\left(i: H^{\ell}\left(\hbar^{i} \overline{\mathcal{R}}_{\hbar}, d_{\hbar}\right) \rightarrow H^{\ell}\left(\overline{\mathcal{R}}_{\hbar}^{\ell}, d_{\hbar}\right)\right)$. Then there are canonical isomorphisms of $\mathrm{k}[\hbar]$-modules

$$
F_{i} H_{\hbar}^{0} / F_{i+1} H_{\hbar}^{0} \stackrel{\sim}{\rightarrow} \hbar^{i} H^{0}\left(\mathcal{R}^{\bullet}, d_{0}\right)
$$

(ii) the same statement as in (i), for $\mathcal{R}_{[[\hbar]]}^{\bullet}=\mathcal{R}^{\bullet}[[\hbar]]$, and

$$
d_{\hbar}^{(\ell)}=d_{0}^{(\ell)}+\hbar d_{1}^{(\ell)}+\hbar^{2} d_{2}^{(\ell)}+\cdots
$$

(possibly an infinite sum), and $d_{\hbar}^{(-1)} \circ d_{\hbar}^{(-2)}=0$.

Proof. The proof is literally the same as for Lemma 2.2, as the only thing we used in it is the vanishing of $H^{-1}\left(\mathcal{R}^{\bullet}, d_{0}\right)$. Consider the short exact sequence 
of complexes:

$$
0 \rightarrow \hbar^{k+1} \overline{\mathcal{R}}_{\hbar} \rightarrow \hbar^{k} \overline{\mathcal{R}}_{\hbar} \rightarrow \hbar^{k} \overline{\mathcal{R}}_{\hbar} / \hbar^{k+1} \overline{\mathcal{R}}_{\hbar} \rightarrow 0
$$

Consider the rightmost fragment of the corresponding long exact sequence in cohomology:

$$
\begin{aligned}
& \cdots \rightarrow H^{1}\left(\hbar^{k+1} \overline{\mathcal{R}}_{\hbar}\right) \rightarrow H^{1}\left(\hbar^{k} \overline{\mathcal{R}}_{\hbar}\right) \rightarrow 0 \rightarrow \\
& \rightarrow H^{0}\left(\hbar^{k+1} \overline{\mathcal{R}}_{\hbar}\right) \rightarrow H^{0}\left(\hbar^{k} \overline{\mathcal{R}}_{\hbar}\right) \rightarrow H^{0}\left(\hbar^{k} \overline{\mathcal{R}}_{\hbar} / \hbar^{k+1} \overline{\mathcal{R}}_{\hbar}\right) \rightarrow 0 .
\end{aligned}
$$

In degrees $\ell=0,-1,-2, \overline{\mathcal{R}}_{\hbar}^{\ell}=\mathcal{R}_{\hbar}^{\ell}$. As well, in degree $\ell=-1$ one has

$$
H^{-1}\left(\hbar^{k} \overline{\mathcal{R}}_{\hbar} / \hbar^{k+1} \overline{\mathcal{R}}_{\hbar}\right)=H^{-1}\left(\hbar^{k} \mathcal{R}_{\hbar}^{\cdot} / \hbar^{k+1} \mathcal{R}_{\hbar}^{\bullet}\right)=0
$$

for any $k \geq 0$, by the assumption. (It is not true for $\ell \leq-2$ ). We are done.

\subsection{Conclusion}

Let

$$
A=T(V)[\hbar] /\left(x_{i} \otimes x_{j}-x_{j} \otimes x_{i}-\varphi_{i j}\right)_{1 \leq i<j \leq n}
$$

be an associative algebra, with $\varphi_{i j} \in \hbar T(V)[\hbar]$.

Consider the Koszul resolution (2.2) of the polynomial algebra $S(V)$, denote it $\mathcal{R}^{\bullet}$. We can perturb the $\mathrm{k}[\hbar]$-linear differential in $\mathcal{R}_{\hbar}^{\bullet}=\mathcal{R}^{\bullet}[\hbar]$ using the non-commutative polynomials $\varphi_{i j}$.

More precisely, we set

$$
d_{\hbar}^{(-1)}\left(\xi_{i j}\right)=d_{0}^{(-1)}\left(\xi_{i j}\right)-\varphi_{i j}=x_{i} \otimes x_{j}-x_{j} \otimes x_{i}-\varphi_{i j}
$$

As $\varphi_{i j}=\mathcal{O}(\hbar)$, we are in the setting of Theorem 2.3. The only thing we need to prove is that the condition of Definition 1.5 is fulfilled for $A$, that is, to perturb the differential component $d^{(-2)}$

$$
d_{\hbar}^{(-2)}\left(\xi_{i j k}\right)=d_{0}^{(-2)}\left(\xi_{i j k}\right)+\mathcal{O}(\hbar)
$$

such that

$$
d_{\hbar}^{(-1)} \circ d_{\hbar}^{(-2)}\left(\xi_{i j k}\right)=0
$$

for any $i, j, k$. 
We may not care about the higher components of the differential, in the sense that we do not need to perturb them.

If we succeed to construct, for given $\left\{\varphi_{i j}\right\}$, the linear map $d_{\hbar}^{(-2)}$ such that (2.20) holds for any $i, j, k$, we know that Definition 1.5 is fulfilled for the algebra $A$ in (2.17), and we can apply Theorem 1.6 to it.

We consider three examples of how it works in Section 3.

\section{Examples}

\subsection{The classical PBW theorem}

Let $\mathfrak{g}$ be a Lie algebra over a field $k$. Consider the cocommutative coalgebra $\Lambda^{-}(\mathfrak{g}):=S^{+}(\mathfrak{g}[1])$. The Lie algebra structure on $\mathfrak{g}$ defines the ChevalleyEilenberg differential on $\Lambda^{-}(\mathfrak{g})$, which makes it a dg coalgebra over $\mathrm{k}$.

There is the $\mathrm{k}[\hbar]$-linear version of this construction. Consider $\Lambda_{\hbar}^{-}(\mathfrak{g}):=$ $\Lambda^{-}(\mathfrak{g})[\hbar]$, as a dg coalgebra. The coproduct $\Delta: \Lambda_{\hbar}^{-}(\mathfrak{g}) \rightarrow S_{\mathrm{k}[\hbar]}^{2} \Lambda_{\hbar}^{-}(\mathfrak{g})$ is defined from the coproduct on $\Lambda^{-}(\mathfrak{g})$ by $\mathrm{k}[\hbar]$-linearity. The differential is defined as $d=\hbar d_{\mathfrak{g}}$ where $d_{\mathfrak{g}}$ is the Chevalley-Eilenberg differential in $\Lambda^{-}(\mathfrak{g})$.

We can now take the tensor algebra over $\mathrm{k}[\hbar] T_{\mathrm{k}[\hbar]}\left(\Lambda^{-} \hbar(\mathfrak{g})[-1]\right)$, it becomes a dg associative algebra. Namely, the differential is the sum $d_{\hbar}=d_{0}+\hbar d_{1}$, where $d_{0}$ is defined in (2.3) above, and $d_{1}$ is the Chevalley-Eilenberg differential (extended by the Leibniz rule).

The identity $\left(d_{0}+\hbar d_{1}\right)^{2}=0$ follows from the fact that $\Lambda_{\hbar}^{-}(\mathfrak{g})$ is an $\mathrm{k}[\hbar]-$ linear dg coalgebra, as it just was defined.

This construction gives the perturbation of the differential in the Koszul resolution $T(S(\mathfrak{g}))[\hbar]$, defined at once in all degrees. In particular,

$$
\begin{aligned}
d^{-1}\left(\xi_{i j}\right) & =x_{i} \otimes x_{j}-x_{j} \otimes x_{i}-\hbar\left[x_{i}, x_{j}\right]_{\mathfrak{g}} \\
d^{-2}\left(\xi_{i j k}\right) & =\operatorname{Cycl}_{i j k}\left(x_{i} \otimes \xi_{j k}-\xi_{j k} \otimes x_{i}\right)-\hbar \sum_{p} \operatorname{Cycl}_{i j k} c_{i j}^{p} \xi_{p k}
\end{aligned}
$$

We conclude that the condition of Definition 1.5 is fulfilled for the algebra $A$ of type (1.1) with

$$
\varphi_{i j}=\hbar\left[x_{i}, x_{j}\right]_{\mathfrak{g}}=\hbar \sum_{k} c_{i j}^{k} x_{k}
$$

This algebra $A$ is, by definition, the quotient-algebra

$$
A=T(\mathfrak{g})[\hbar] /\left(x \otimes y-y \otimes x-\hbar[x, y]_{\mathfrak{g}}\right)_{x, y \in \mathfrak{g}}=\mathcal{U}\left(\mathfrak{g}_{\hbar}\right)[\hbar]
$$


To apply Theorem 1.6(i), we need the following elementary Lemma:

Lemma 3.1. Let $A$ be an algebra of type (1.1) such that all $\varphi_{i j}$ be linear in $\left\{x_{i}\right\}$. Then $\bigcap_{\ell \geq 0} \Gamma_{\ell}(A)=0$.

Proof. It is clear.

Now Theorem 1.6(i) gives the classical PBW theorem (in its $\mathrm{k}[\hbar]$-linear version). We can specialize it at any $\hbar=a$ as $F_{k}(A) / F_{k-1}(A) \simeq S^{k}(V)[\hbar]$ is a free (and therefore a flat) $\mathrm{k}[\hbar]$-module. It gives the genuine $\mathrm{PBW}$ theorem.

\subsection{The Etingof-Ginzburg algebras with cyclic cubic potential}

Recall some basic definitions on cyclic words.

A cyclic polynomial in variables $x_{1}, \ldots, x_{n}$ is an element of the quotientspace $A_{n} /\left[A_{n}, A_{n}\right]$, where $A_{n}=T\left(x_{1}, \ldots, x_{n}\right)$ is the free associative algebra with generators $x_{1}, \ldots, x_{n}$. A homogeneous of degree $d$ cyclic polynomial in $x_{1}, \ldots, x_{n}$ is an element of degree $d$ component in $A_{n} /\left[A_{n}, A_{n}\right]$, where the degree of all $x_{i}$ is equal to 1 .

Let $\Phi$ be a cyclic polynomial in $x_{1}, \ldots, x_{n}$. Here we define the partial derivatives $\frac{\partial \Phi}{\partial x_{i}}$. These derivatives are not cyclic polynomials, but elements of $A_{n}=T\left(x_{1}, \ldots, x_{n}\right)$. By definition, for a single cyclic monomial $\Phi, \frac{\partial \Phi}{\partial x_{i}}$ is a sum over all occurrences of $x_{i}$ in $\Phi$, for any such occurrence, we remove the corresponding $x_{i}$ from $\Phi$, and cut off the cyclic word $\Phi$ in the place of removed $x_{i}$. Then extend it to general cyclic polynomials by linearity. One can easily see that the partial derivative $\frac{\partial}{\partial x_{i}}$ vanishes on the commutator $\left[A_{n}, A_{n}\right]$, and defines an operation

$$
\frac{\partial}{\partial x_{i}}: A_{n} /\left[A_{n}, A_{n}\right] \rightarrow A_{n} .
$$

Let $\Phi$ be an arbitrary cyclic monomial in $A=A_{3}=T(x, y, z)$. The Etingof-Ginzburg algebra with cyclic potential $\Phi$ is the associative algebra with generators $x, y, z$ and the defining relations

$$
\begin{aligned}
& x \otimes y-y \otimes x=\frac{\partial \Phi}{\partial z}, \\
& y \otimes z-z \otimes y=\frac{\partial \Phi}{\partial x}, \\
& z \otimes x-x \otimes z=\frac{\partial \Phi}{\partial y} .
\end{aligned}
$$

We use the $[\mathrm{EG}]$ notation $\mathcal{U}(\Phi)$ for these algebras. 
The associative algebras $\mathcal{U}(\Phi)$ appeared in [EG] in constructing of "noncommutative del pezzo surfaces" (loc.cit., Sections 1.2-1.3, see also [G]).

Let $\hbar$ be a parameter. Consider an expression

$$
\Phi_{\hbar}=\hbar \Phi_{1}+\hbar^{2} \Phi_{2}+\cdots
$$

which is a polynomial in $\hbar$, all whose coefficients $\Phi_{i}$ are possibly nonhomogeneous cyclic words (without any restriction on the degrees of homogeneity components) in $x, y, z$.

Define the $\mathrm{k}[\hbar]$-linear associative algebra $\mathcal{U}_{\hbar}\left(\Phi_{\hbar}\right)$ as the quotient-algebra of the $\mathrm{k}[\hbar]$-linear tensor algebra $T(x, y, z)[\hbar]$ by the two-sided ideal, generated by the following three elements:

$$
\begin{aligned}
& {[x, y]-\frac{\partial \Phi_{\hbar}}{\partial z}} \\
& {[y, z]-\frac{\partial \Phi_{\hbar}}{\partial x}} \\
& {[z, x]-\frac{\partial \Phi_{\hbar}}{\partial y}}
\end{aligned}
$$

Lemma 3.2. Let $\Phi_{\hbar}$ be as in (3.4). Then the associative algebra $\mathcal{U}_{\hbar}\left(\Phi_{\hbar}\right)$ enjoys the $P B W$ property for the descending filtration $\left\{\Gamma_{\ell}\right\}$ of Definition (1.5).

Proof. Accordingly to Theorem 2.3 and discussion in Section 2.4 thereafter, it is enough to perturb the $(-2)$-component of the differential in the Koszul resolution $\mathcal{R}^{\bullet}\left(x_{1}, x_{2}, x_{2}\right)$ such that the perturbation of the component $d^{(-1)}$ is

$$
d_{\hbar}^{(-1)}\left(\xi_{i j}\right)=x_{i} \otimes x_{j}-x_{j} \otimes x_{i}-\frac{\partial}{\partial x_{\overline{i, j}}} \Phi_{\hbar},
$$

where we use the notation $\overline{1,2}=3, \overline{2,3}=1, \overline{3,1}=2$, and assume that $x_{\overline{i, j}}=-x_{\overline{j, i}}$.

That is, the only what we need is to define a perturbation $d_{\hbar}^{(-2)}\left(\xi_{i j k}\right)$ such that

$$
d_{\hbar}^{(-1)} \circ d_{\hbar}^{(-2)}\left(\xi_{i j k}\right)=0
$$

Our solution is do not perturb $d^{(-2)}$ at all. That is, we set

$$
d_{\hbar}\left(\xi_{123}\right)=d_{0}\left(\xi_{123}\right)=\left[\xi_{12}, x_{3}\right]+\left[\xi_{23}, x_{1}\right]-\left[\xi_{13}, x_{2}\right],
$$


We need to check that this definition agrees with (3.7).

Lemma 3.3. Within the definitions (3.6) and (3.8), one has $d_{\hbar}^{2}\left(\xi_{123}\right)=0$.

Proof. It is easy to see that

$$
d_{\hbar}^{2}\left(\xi_{123}\right)=\left[\frac{\partial \Phi_{\hbar}}{\partial x_{1}}, x_{1}\right]+\left[\frac{\partial \Phi_{\hbar}}{\partial x_{2}}, x_{2}\right]+\left[\frac{\partial \Phi_{\hbar}}{\partial x_{3}}, x_{3}\right] .
$$

We claim that the sum in the r.h.s. is 0 (for any cyclic word $\Phi_{\hbar}$ ).

Indeed, the first "half" of the sum in the r.h.s. of (3.9),

$$
\frac{\partial \Phi_{\hbar}}{\partial x_{1}} \otimes x_{1}+\frac{\partial \Phi_{\hbar}}{\partial x_{2}} \otimes x_{2}+\frac{\partial \Phi_{\hbar}}{\partial x_{3}} \otimes x_{3}
$$

is equal to the sum of all possible cuttings (at any place) of the cyclic word $\Phi_{\hbar}$ to an ordinary word.

The remaining second "half" of the r.h.s. of (3.9),

$$
-x_{1} \otimes \frac{\partial \Phi_{d}}{\partial x_{1}}-x_{2} \otimes \frac{\partial \Phi_{d}}{\partial x_{2}}-x_{3} \otimes \frac{\partial \Phi_{d}}{\partial x_{3}}
$$

is equal to the same sum of all possible cuttings of the cyclic word $\Phi_{d}$ to an ordinary word, taken with the opposite sign.

The contributions of (3.10) and (3.11) cancel each other.

Now Lemma 3.2 follows directly from Theorem 2.3.

Theorem 1.6(ii) gives now the following result.

Corollary 3.4. Let $\Phi_{\hbar}$ be as in (3.4) with $\operatorname{deg}_{\left\{x_{i}\right\}} \Phi_{\hbar} \leq 3$, and let $A=$ $\mathcal{U}_{\hbar}\left(\Phi_{\hbar}\right)$. Then for all but countably many $a \in \mathrm{k}$ the specialization $A_{a}$ is a $P B W$ algebra in the sense of Definition 1.3, and for all such $a \in \mathrm{k}$ is the specialized algebra is isomorphic to $\mathcal{U}(\Phi(a))$, where $\Phi(a)$ is the value of $\Phi(\hbar)$ at $\hbar=a$.

We conclude the discussion of Etingof-Ginzburg algebras by an explicit computation, illustrating the assumption in Theorem 1.6(ii): $\bigcap_{\ell} \Gamma_{\ell}$ $(A) \neq 0$.

3.2.1. Example. Consider $\Phi_{\hbar}=\hbar \operatorname{Cycl}(-z y x)$ where $\operatorname{Cycl}(-)$ denotes the cyclic word associated to a non-commutative monomial. Then the algebra 
$\mathcal{U}_{\hbar}^{\mathrm{pol}}\left(\Phi_{\hbar}\right)$ has the following relations:

$$
\begin{aligned}
& {[x, y]=-\hbar y x,} \\
& {[y, z]=-\hbar z y,} \\
& {[z, x]=-\hbar x z .}
\end{aligned}
$$

Its specialization at $\hbar=1$ gives the associative algebra with relations:

$$
\begin{aligned}
& x y=0, \\
& y z=0, \\
& z x=0 .
\end{aligned}
$$

The basis of this algebra as a $k$-vector space is formed by the monomials:

$$
x^{m_{1}} z^{m_{2}} y^{m_{3}} x^{m_{4}} z^{m_{5}} y^{m_{6}} \ldots
$$

with all $m_{i} \geq 0$. It is clear that the graded components of this algebra are much bigger than the corresponding graded components for $S(x, y, z)$. That is, this algebra is not a PBW algebra.

It follows from Theorem 1.6(i) that for $\Phi_{\hbar}=-\hbar z y x$ one should have

$$
I\left(\Phi_{\hbar}\right)=\bigcap_{\ell=0}^{\infty} \Gamma_{\ell} \mathcal{U}_{\hbar}\left(\Phi_{\hbar}\right) \neq 0 .
$$

Recall the Krull intersection theorem:

Theorem 3.5 (Krull). Let $A$ be a noetherian commutative ring, $\mathfrak{q}$ an ideal of $A$, and $M$ a finitely generated $A$-module. Then $m \in \cap_{n} \mathfrak{q}^{n} M$ if and only if there exists $d \in \mathfrak{q}$ such that $d m=m$. In particular, if $\cap_{n} \mathfrak{q}^{n} M \neq 0$, there exist $0 \neq m \in M$ and $d \in \mathfrak{q}$ such that $d m=m$.

See, e.g., [M, Theorem 8.9] for a proof.

For some non-commutative rings, the analogous result still holds; see $[\mathrm{MC}]$.

We can apply the result from loc.cit. to $A=\mathcal{U}_{\hbar}\left(\Phi_{\hbar}\right)$, for $\Phi_{\hbar}=-\hbar z y x$, and for $\mathfrak{q}=\hbar \mathfrak{U}_{\hbar}\left(\Phi_{\hbar}\right), M=A$. It is instructive to find explicitly an element $m \in \mathcal{U}_{\hbar}\left(\Phi_{\hbar}\right)$ in the intersection $\bigcap_{\ell \geq 0} \Gamma_{\ell}(A)$. It turns out that such $m$ can be found cubic in $x, y, z$, with $d=\hbar \cdot \overline{1}$.

For this end, consider equations (3.12). We can multiply the first among them by $z$ from the left and from the right (and thus we get two equations), 
multiply the second one by $x$ from the left and from the right, and multiply the third one by $y$ from the left and from the right. Overall, we get six equations listed below.

$$
\begin{aligned}
& \text { (A1) } \quad z x y-z y x=-\hbar z y x \\
& \text { (C1) } \quad x y z-y x z=-\hbar y x z, \\
& \text { (C2) } \quad x y z-x z y=-\hbar x z y, \\
& \text { (B1) } \quad y z x-z y x=-\hbar z y x \\
& \text { (B2) } \quad y z x-y x z=-\hbar y x z, \\
& \text { (A2) } \quad z x y-x z y=-\hbar x z y .
\end{aligned}
$$

We group these six equations in three groups, labeled A, B and C. Each group contains two equations, having equal first summands in the 1.h.s. Thus, the lhs of (A1) and (A2) have equal to zxy first summands.

Now we consider three new equations, obtained from these six equations as $(\mathrm{A} 1)-(\mathrm{A} 2),(\mathrm{B} 1)-(\mathrm{B} 2),(\mathrm{C} 1)-(\mathrm{C} 2)$. They are

$$
\begin{aligned}
& \text { (A) }-z y x+x z y=\hbar(-z y x+x z y), \\
& \text { (B) }-z y x+y x z=\hbar(-z y x+y x z), \\
& \text { (C) }-y x z+x z y=\hbar(-y x z+x z y)
\end{aligned}
$$

(notice that there is 1 linear dependence between these three equations).

Each of these three lines has form $T=\hbar \cdot T$ for some cubic $T$. Then we have:

$$
T=\hbar \cdot T=\hbar^{2} \cdot T=\hbar^{3} \cdot T=\cdots,
$$

which shows that each such $T$ belongs to $\bigcap_{\ell \geq 0} \Gamma_{\ell}\left(\mathcal{U}_{\hbar}\left(\Phi_{\hbar}\right)\right)$.

Notice that any such $T$ is killed under the completion homomorphism, as the equation $(\hbar-1) T=0$, under formal power series in $\hbar$, implies $T=0$, as $\hbar-1$ becomes invertible.

\section{The case of general quadratic algebras}

\section{1.}

Suppose we are given an associative algebra of type (1.1) with quadratic $\varphi_{i j}$. Here we find a criterion under which this algebra enjoys the property of Definition 1.5, which guarantees that it is a PBW algebra in the sense of Definition 1.3, for generic specialization of $\hbar=a \in \mathrm{k}$. 
Let

$$
\varphi_{i j}=\hbar \sum_{a, b} \alpha_{i j}^{a b} x_{a} \otimes x_{b}
$$

where

$$
\alpha_{i j}^{a b}=-\alpha_{j i}^{a b} \text {, but } \alpha_{i j}^{a b} \neq \alpha_{i j}^{b a} \text {, in general. }
$$

Our main result here is

Theorem 4.1. In the notations as above, suppose that for $\forall i, j, k, b, c, d$ :

$$
\mathrm{Cycl}_{i, j, k} \sum_{s}\left(\alpha_{j k}^{s b} \alpha_{i s}^{c d}+\alpha_{j k}^{c s} \alpha_{i s}^{d b}\right)=0 .
$$

Then the associative algebra $A$ in (1.1) enjoys the PBW-like property of Definition (1.5) for the descending filtration $\left\{\Gamma_{\ell}\right\}$, and the genuine $P B W$ property of Definition 1.3 for generic specialization $\hbar=a$.

Remark 4.2. Let $\left\{\alpha_{i j}^{a b}\right\}$ satisfy (4.3). Let $\beta=\beta_{i j} \partial_{i} \wedge \partial_{j}, \beta_{i j}=\sum_{a, b}\left(\alpha_{i j}^{a b}+\right.$ $\left.\alpha_{i j}^{b a}\right) x_{a} x_{b}$, be the corresponding bivector on $V=\left\{x_{1}, \ldots, x_{n}\right\}$. Then the bivector $\beta$ is Poisson: in coordinates, for any $i, j, k, a, b, c$ :

$$
\sum_{s}\left(\beta_{i s}^{a b} \beta_{j k}^{s c}+\beta_{j s}^{a b} \beta_{k i}^{s c}+\beta_{k s}^{a b} \beta_{i j}^{s c}\right)=0 .
$$

Indeed, (4.4) is obtained from (4.3) by symmetrization in the upper indices. It is very natural, as the Poisson condition is equivalent to the flatness in degree 2 in $x_{i}$ 's.

Proof. We prove the first claim, that the corresponding algebra $A$ enjoys the property of Definition 1.5. The last claim follows then immediately from Theorem 1.6(ii).

By Section 2.4 and Theorem 2.3, we need to perturb the (-2)-component of the differential $d_{\hbar}^{(-2)}\left(\xi_{i j k}\right)$, such that

$$
d_{\hbar}^{(-1)}\left(\xi_{i j}\right)=x_{i} \otimes x_{j}-x_{j} \otimes x_{i}-\varphi_{i j}
$$

where $\varphi_{i j}$ is as in (4.1). Then the only property to be checked is

$$
d_{\hbar}^{(-1)} \circ d_{\hbar}^{(-2)}\left(\xi_{i j k}\right)=0
$$

for any $i, j, k$. 
We define

$$
d_{\hbar}^{(-2)}\left(\xi_{i j k}\right)=\operatorname{Cycl}_{i, j, k}\left[x_{i}, \xi_{j k}\right]+\hbar \operatorname{Cycl}_{i, j, k} \sum_{a, b} \alpha_{j k}^{a b}\left(\xi_{i a} \otimes x_{b}+x_{a} \otimes \xi_{i b}\right)
$$

Then (4.6) has the terms of order $0,1,2$ in $\hbar$. The cancelation of $\hbar^{0}$ terms is just the condition $d^{2}=0$ for the non-perturbed differential. The cancelation in order $\hbar^{1}$ is straightforward and does not use (4.3); in fact, the formula (4.7) was designed especially to maintain this cancelation.

We need only to check (4.6) in order $\hbar^{2}$ then. We have:

$$
d_{\hbar}^{2}\left(\xi_{i j k}\right)=\hbar^{2} \operatorname{Cycl}_{i j k} \sum_{a b c d}\left(\alpha_{j k}^{a b} \alpha_{i a}^{c d} x_{c} \otimes x_{d} \otimes x_{b}+\alpha_{j k}^{a b} \alpha_{i b}^{c d} x_{a} \otimes x_{c} \otimes x_{d}\right)
$$

To satisfy (4.6), the coefficient at $x_{a} \otimes x_{b} \otimes x_{c}$ for any $a, b, c$ (and for any $i, j, k)$ should vanish. It gives our condition (4.3).

Remark 4.3. For an algebra $A$ of type (1.1), one has

$$
\left[x_{i}, x_{j}\right]=\hbar \varphi_{i j}
$$

where by $x_{i}$ 's and by $\varphi_{i j}$ is meant their image in the quotient-algebra $T(V)[\hbar] / \mathcal{J}$. The Jacobi identity

$$
\mathrm{Cycl}_{i, j, k}\left[x_{i},\left[x_{j}, x_{k}\right]\right]=0
$$

holds in any associative algebra. Using (4.9) twice, it gives

$$
\overline{\mathrm{Cycl}_{i j k} \sum_{a, b, c, d}\left(\alpha_{j k}^{a b} \alpha_{i a}^{c d} x_{c} \otimes x_{d} \otimes x_{b}+\alpha_{j k}^{a b} \alpha_{i b}^{c d} x_{a} \otimes x_{c} \otimes x_{d}\right)}=0
$$

if

$$
\varphi_{i j}=\hbar \sum_{a, b} \alpha_{i j}^{a b} x_{a} \otimes x_{b}
$$

where $\bar{\omega}$ for an $\omega \in T(V)[\hbar]$ stands for its image in the quotient-algebra $A=T(V)[\hbar] / \mathcal{J}$.

That is, (4.11) holds for any $\left\{\varphi_{i j}\right\}$. Our condition (4.3) is exactly (4.11) without the overline sign. 
That is, it says that not only the image $\bar{\omega}$ of

$$
\omega=\mathrm{Cycl}_{i j k} \sum_{a, b, c, d}\left(\alpha_{j k}^{a b} \alpha_{i a}^{c d} x_{c} \otimes x_{d} \otimes x_{b}+\alpha_{j k}^{a b} \alpha_{i b}^{c d} x_{a} \otimes x_{c} \otimes x_{d}\right)
$$

vanishes in the quotient-algebra $T(V)[\hbar] / \mathcal{J}$ but, essentially stronger, $\omega$ itself is equal to zero in $T(V)[\hbar]$.

The Etingof-Ginzburg algebras do not obey, in general, the condition (4.3). Seemingly, the case of dimension 3 is very special for examples of PBW algebras, due to some "additional symmetries" in dimension 3 . When we are interested in PBW algebras defined over polynomials $\mathrm{k}[\hbar]$, this symmetry makes possible the existence of some "irregular" examples.

See Section 0.4 of the Introduction for comparison with Drinfeld's result $[\mathrm{D}]$.

\section{Acknowledgements}

I am grateful to Pavel Etingof, Boris Feigin, Victor Ginzburg, Volodya Rubtsov, Matthew Tucker-Simmons, Michel Van den Bergh, and Vadik Vologodsky, for a fruitful correspondence/discussions. Volodya Rubtsov's many questions on [Sh2] stimulated my further work on it, in an attempt to answer them. Then I found a new proof of PBW property for Etingof-Ginzburg algebras, following that method. Pavel Etingof was the very first I communicated the proof to, and his inspiration was another good stimulus for continuation of this work. Many of Pavel's explanations and remarks were very helpful as well.

I am grateful to the anonymous referee whose careful reading of the manuscript and numerous remarks and corrections have led to thorough improvements in the revised version.

This work as [Sh2] was started in Luxembourg around 2007. I am thankful to the University of Luxembourg and personally to Martin Schlichenmaier, for excellent working conditions and for financial support, under the research grant R1F105L15 of the University of Luxembourg.

The work was completed in 2013-2014 in the University of Antwerp, with partial financial support by FWO "Krediet aan navorser" grant. 


\section{References}

[BG] A. Braverman and D. Gaitsgory, Poincare-Birkhoff-Witt theorem for quadratic algebras of Koszul type, J. Algebra, 181 (1996), 315-328.

[BGS] A. Beilinson, V. Ginzburg and W. Soergel, Koszul duality patterns in representation theory, J. AMS, 9(2) (1996), 473-527.

[D] V. Drinfeld, On quadratic commutation relations in the quasiclassical case, Select. Math. Sov., 11(4) (1992), 317-326.

[EG] P. Etingof and V. Ginzburg, Noncommutative Del Pezzo surfaces and Calabi-Yau algebras, arXiv: math.0709.3593.

[G] V. Ginzburg, Calabi-Yau algebras, arXiv: math.0612139.

[M] H. Matsumura, Commutative ring theory, Cambridge Studies in Advanced Mathematics 8, Cambridge University Press, 1986.

[MC] J.C. McConnell, The intersection theorem for a class of noncommutative rings, Proc. London Math. Soc., 17(3) (1967), 487-498

[PP] A. Polishchuk and L. Positselski, Quadratic Algebras, AMS University Lecture Series, 2005.

[Sh1] B. Shoikhet, Koszul duality in deformation quantization and Tamarkin's approach to Kontsevich formality, Adv. Math., 224 (2010), 731-771.

[Sh2] B. Shoikhet, PBW algebras and Kontsevich formality, arXiv: math.0708.1634.

UNIVERSITEIT ANTWERPEN

Campus Middelheim, Wiskunde en Informatica, Gebouw G

MiddelheimlaAn 1, 2020 Antwerpen

BELGIUM

E-mail address: Boris.Shoikhet@uantwerpen.be

Received OCTOBer 31, 2013 